\title{
A casein-based diet leads to a better bone status than a soy protein-based diet during moderate protein restriction in growing mice
}

AgroParisTech

$\mathrm{BTO}$

\author{
Rouy E. ${ }^{1,2}$, Laroche N. ${ }^{3}$, Blachier F. ${ }^{1}$, Tomé D. ${ }^{1}$, Vico L. ${ }^{3}$ and Blais A. ${ }^{1}$ \\ Contact : emilien.rouy@agroparistech.fr
}

${ }^{1}$ UMR 914 PNCA, Paris, France ; ${ }^{2}$ Yoplait France ; ${ }^{3}$ U1059 LBTO, Saint Etienne, France

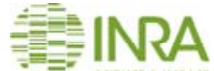

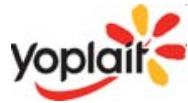

\section{Background}

The peak bone mass reached during childhood and adolescence strongly influences bone status in old age. An optimal bone acquisition during growth can be perceived as a mean to reduce fracture risk in later life. In animal models, it has been shown that insufficient protein intake during early stages of life leads to growth retardation and poor bone quality.

Our aim was to compare the effects of a casein-based diet and a soy-based diet on bone acquisition during moderate protein restriction ( $6 \%$ of energy intake) in a growing mouse model.

\section{Protocol}

\begin{tabular}{|c|c|c|}
\hline Group & Diet & Injection \\
\hline LP-SOY & $6 \%$ soy protein & PBS \\
\hline LP-CAS & $6 \%$ casein & PBS \\
\hline NP & $20 \%$ soy protein & PBS \\
\hline PTH & $6 \%$ soy protein & PTH 1-34 \\
\hline
\end{tabular}

\section{Results}

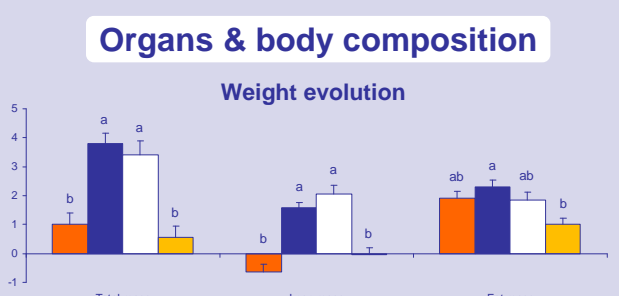

Brown adipose tissue weight

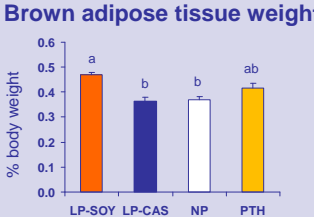

Uterine weight

Pese increased brown adipose tissue weight when compared to NP and LP-CAS diets.

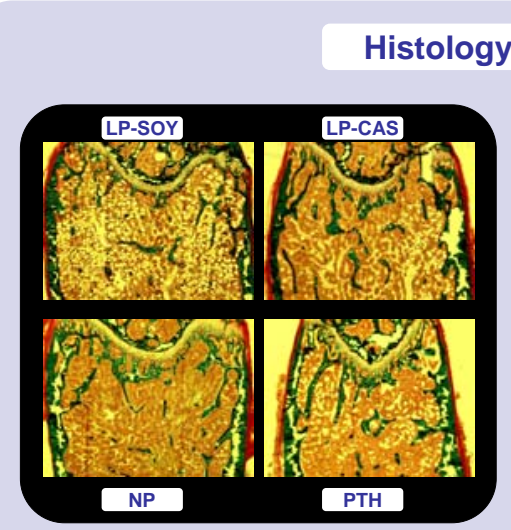

Osteoclast surface

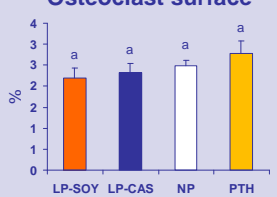

Osteoid surface

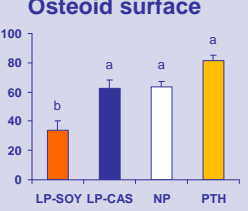

Adipocyte volume

${ }^{10}$

6 -

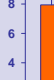

2.

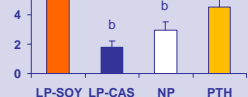

Bone formation rate

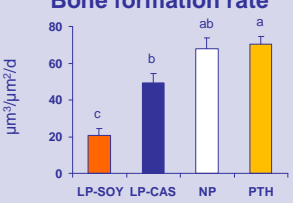

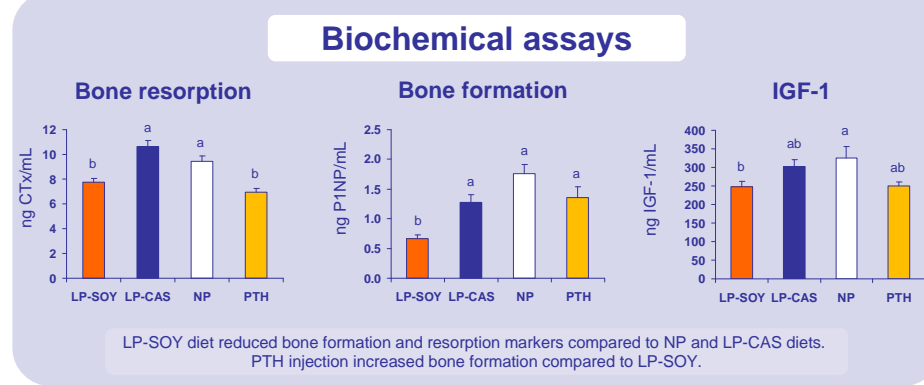

\section{Bone microarchitecture}

\section{Cortical bone}
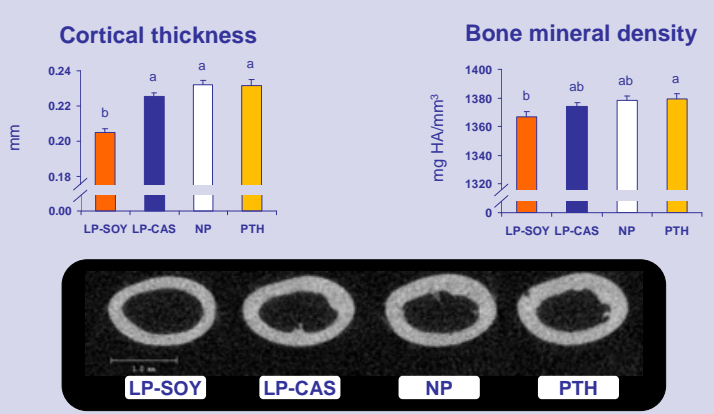

LP-SOY reduced cortical thickness compared to NP and LP-CAS diets.

Trabecular bone

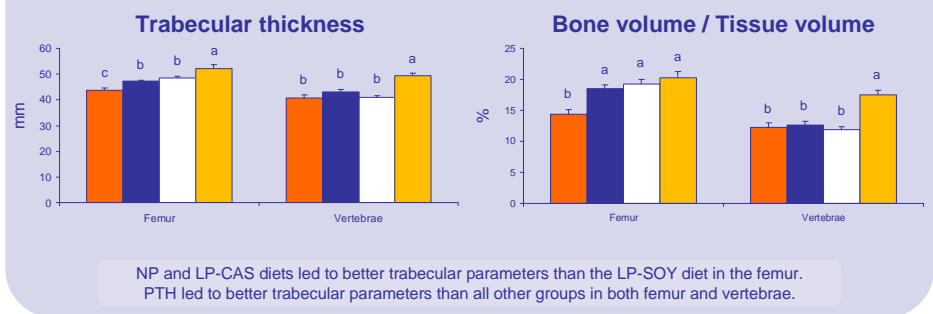

\section{Conclusions}

\section{The main conclusions are}

- A soy-based moderate protein restriction reduced bone formation and lowered bone quality (LP-SOY vs NP)

- Under protein restriction, a casein diet resulted in better bone quality than a soy diet due to reduced bone formation (LP-SOY vS LP-CAS)

- The study also showed that daily PTH 1-34 injection had an anabolic effect under protein restriction (LP-SOY vs PTH)
Our results show that both NP and LP-CAS diets are more suitable than LP-SOY diet to ensure an adequate bone health during growth.The LPSOY diet is characterized by a reduction of bone formation leading to poor bone quality when compared to the NP diet or the LP-CAS diet.

Our hypothesis is that bone formation is reduced when a critically low level is reached for one or several amino acids, which could at least partly explain the observed effects. Methionine could be the trigger amino acid as soy contains less methionine than casein. 